\title{
THE ENERGY DEPENDENCE OF SINGLE PARTICLE SPECTRA IN $\mathrm{e}^{+} \mathrm{e}^{-}$ANNIHILATION
}

\author{
J. ENGELS, H. SATZ and K. SCHILLING \\ Department of Theoretical Physics, University of Bielefeld, Germany
}

Received 11 February 1974

\begin{abstract}
We present explicit predictions of the statistical bootstrap model for inclusive single particle spectra in $\mathrm{e}^{+} \mathrm{e}^{-}$ annihilation. The distribution $\left(\omega / \sigma_{\text {tot }} E\right)\left(\mathrm{d} \sigma / p^{2} \mathrm{~d} p\right)$ is found to besome, for $E \geqslant 3 \mathrm{GeV}$, a function only of the secondary energy $\omega$, independent of the incident $\mathrm{e}^{+} \mathrm{e}^{-}$energy $E$.
\end{abstract}

The statistical bootstrap model $[1,2]$ applied to $\mathrm{e}^{+} \mathrm{e}^{-}$annihilation into hadrons [3] leads to predictions which are quite striking and at the same time unambiguous, critical tests of the underlying picture: the average energy per secondary is expected to become asymptotically constant, the hadron multiplicity to rise linearly with the mass of the virtual photon (assuming single photon exchange dominance). These predictions appear to be specific of the statistical bootstrap model and related considerations [4]; they are quite in contrast to what is expected from essentially all other theoretical schemes proposed so far [5].

Two further features make the statistical bootstrap model (SBM) a particularly attractive candidate to be tested by $\mathrm{e}^{+} \mathrm{e}^{-}$annihilation experiments presently being carried out. The model was originally developed for hadron-hadron interactions, and the only open parameter, the ultimate temperature $T_{o}$ for hadronic matter at rest, can essentailly be determined from inclusive transverse momentum distributions in high energy hadron-hadron collisions, e.g. from $90^{\circ}$ ISR data [6]. The use of these data not only provides for $\mathrm{e}^{+} \mathrm{e}^{-}$annihilation a parameter-free numerical description; it also predicts a very early onset of asymptotic behaviour $\left(E_{\mathrm{e}^{+} \mathrm{e}^{-}} \geqslant 3 \mathrm{GeV}\right)[3]$.

The aim of the present note is to present in particular the numerical predictions of the SBM for normalized inclusive single particle spectra at various $\mathrm{e}^{+} \mathrm{e}^{-}$energies. These appear to be among the first experimentally accessible quantities, and they allow a test of the distribution both in total incident energy and in the energy of the secondary.

The basis of the SBM is given (a) by the "statistical assumption" that transitions are determined by level densities alone (i.e., that all possible exclusive states contribute equally and with random phases to a given inclusive state), and (b) by the "bootstrap condition" requiring the level density of a hadronic system to be the same as the mass spectrum of its constituents. For further details we refer to ref. [3] and state here only the essential result: the level density $\tau(M)$ for a hadronic system of mass $M$ is given by [7]

$\tau(M)=\sum_{l=1}^{\infty} g_{l} B^{l-1} \int \prod_{i=1}^{l} \frac{\mathrm{d}^{3} p_{i}}{2 p_{i \mathrm{o}}} \delta^{4}\left(\sum_{j=1}^{l} p_{j}-Q\right)_{Q^{2}=M^{2}}$

where the parameter $B$ is determined by the ultimate temperature $T_{\mathrm{o}}$ and the mass $m$ of the secondary

$2 \pi m B k T_{\mathrm{o}} K_{1}\left(m / k T_{\mathrm{o}}\right)=2 \ln 2-1$

( $k$ denotes the Boltzmann constant, $K_{1}$ is the modified Hankel function). The coefficients $g_{1}$ satisfy a "bootstrap" recursion relation [3] which can asymptotically be solved to yield

$\tau(M)=$ const. $M^{-3} \exp \left(M / k T_{\mathrm{o}}\right)$.

Applied to hadron-hadron collisions, this result predicts the asymptotic inclusive transverse momentum spectrum

$\mathrm{d} \sigma / \mathrm{d} p_{\mathrm{T}}^{2} \sim \exp \left(-\sqrt{p_{\mathrm{T}}^{2}+m^{2} / k T_{\mathrm{o}}}\right)$.

If we take the observed ISR behaviour to be fully asymptotic, the $90^{\circ}$ data [6] fix $k T_{\mathrm{o}}=160 \mathrm{MeV}$. If, on the other hand, proton-proton collisions at ISR energies lead to the formation of clusters with not quite asymptotic masses [e.g. 8] $M_{\mathrm{F}}$, then we must perform the (non-asymptotic) calculation, eq. (1), with $M=M_{\mathrm{F}}$ to determine the transverse spectrum. This results in a somewhat higher ultimate temperature $T_{0}$; e.g. with $M_{\mathrm{F}}=2 \mathrm{GeV}$ at ISR energies [8], 
Table 1

\begin{tabular}{lll}
\hline & $k T_{\mathrm{o}}=160 \mathrm{MeV}$ & $k T_{\mathrm{o}}=193 \mathrm{MeV}$ \\
\hline $\bar{\omega}_{\pi}$ & $414 \mathrm{MeV}$ & $475 \mathrm{MeV}$ \\
$\bar{N}_{\pi}$ & $2.42 \cdot E$ & $2.11 \cdot E$ \\
$\frac{2 \omega}{\sigma_{\text {tot }}} \frac{\mathrm{d}^{3} \sigma}{\mathrm{d}^{3} p}$ & $22.87 E \exp (-6.25 \omega)$ & $12.43 E \exp (-5.18 \omega)$ \\
\hline
\end{tabular}

$E$ and $\omega$ inserted in $\mathrm{GeV}$.

we obtain $k T_{\mathrm{o}}=193 \mathrm{MeV}$. It is, however, evident that this uncertainty in hadronic processes allows only a very restricted variation of $T_{0}$; essentially we have

$$
160 \lesssim k T_{\mathrm{o}} \lesssim 200 \mathrm{MeV}
$$

as possible range of the ultimate temperature.

So far we have considered the case with only one type of secondary. If $\nu$ different secondaries are present the corresponding SBM contains $\nu-1$ parameters which determine the relative particle yields. In the numerical calculations, we have used the corresponding $90^{\circ}$ ISR ratio

$\bar{N}_{\pi^{+}}: \bar{N}_{\mathrm{K}^{+}}=100: 7$.

Should considerable deviations from this value occur in $\mathrm{e}^{+} \mathrm{e}^{-}$annihilation, the calculation must be repeated with suitably modified parameters.

With this, the model is completely determined and can now be applied to $\mathrm{e}^{+} \mathrm{e}^{-}$annihilation, where - as is the fundamental feature of all statistical descriptions - it can predict all relative quantities (normalized spectra, multiplicities, average energies, etc.). The absolute value of the total cross section is in principle not predictable by such models.

The essential asymptotic results of the $\mathrm{e}^{+} \mathrm{e}^{-} \mathrm{SBM}$ are summarized in table 1 , where we give the average energy per pion $\bar{\omega}_{\pi}$, pion multiplicity $\bar{N}_{\pi}$, and the normalized invariant spectrum $\left(2 \omega / \sigma_{\text {tot }}\right)\left(\mathrm{d}^{3} \sigma / \mathrm{d}^{3} p\right)$ as a function of the incoming energy $E$ for $k T_{\mathrm{o}}=$ $160 \mathrm{MeV}$ and $193 \mathrm{MeV}$ for $\mathrm{e}^{\dagger} \mathrm{e}^{-} \rightarrow$ pions.

In order to display scaling properties of the process, it is convenient to write the inclusive spectrum in terms of the scaling variable $x=2 \omega / E$. For asymptotic values of $E$, SBM predicts
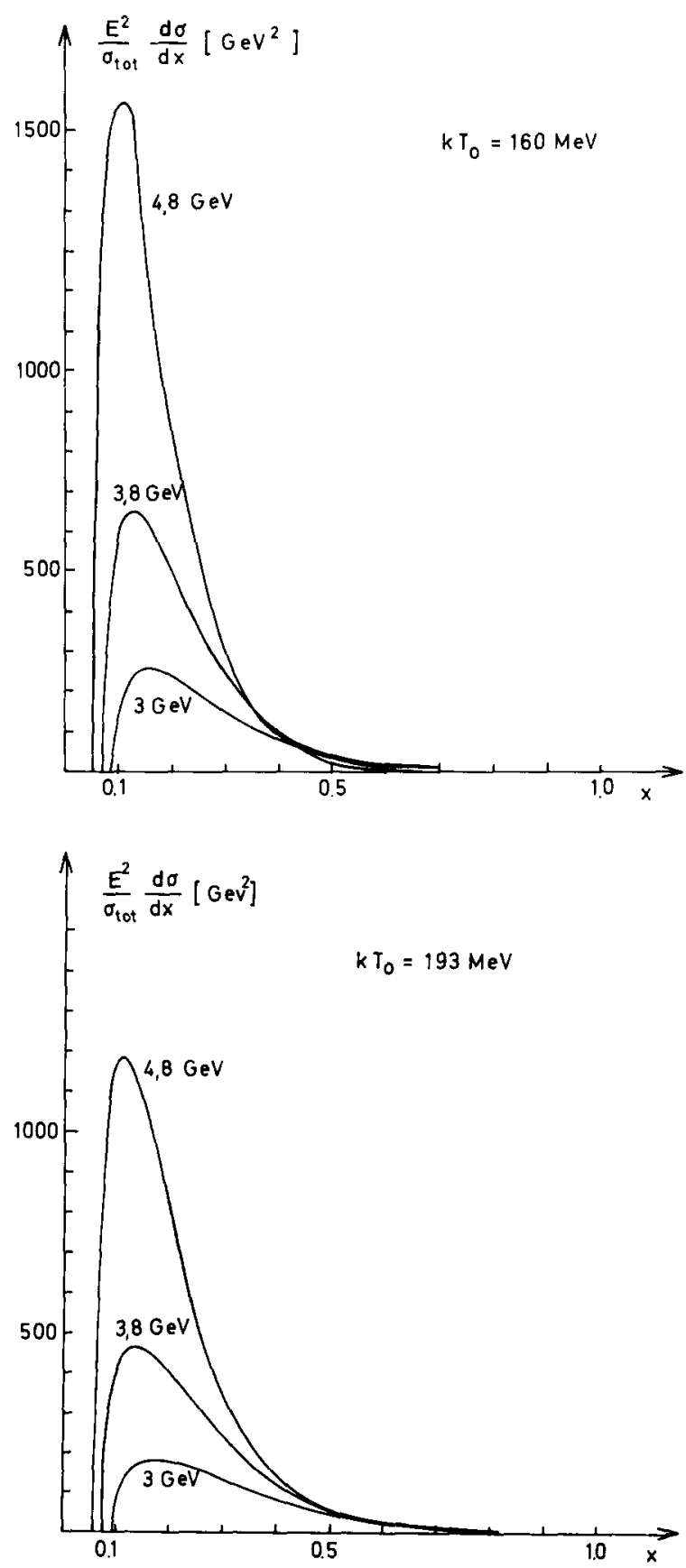

Fig. 1. (a) Asymptotic predictions for $\left(E^{2} / \sigma_{\text {tot }}\right)(\mathrm{d} \sigma / \mathrm{d} x)$ (ete $\mathrm{e}^{-} \rightarrow$ pions (eq. (7)) at $k T_{\mathrm{o}}=160 \mathrm{MeV}$ for $E=2,3.8$ and $4.8 \mathrm{GeV}$. (b) Same as in (a) with $k T_{\mathrm{o}}=193 \mathrm{MeV}$. 
$\frac{E^{2}}{\sigma_{\text {tot }}} \frac{\mathrm{d} \sigma}{\mathrm{d} x}=$

$\frac{E^{5}}{4 m^{2} k T_{\mathrm{o}} K_{2}\left(m / k T_{\mathrm{o}}\right)}\left(1-\frac{4 m^{2}}{(E x)^{2}}\right)^{1 / 2} x \exp \left(-\frac{E x}{2 k T_{\mathrm{o}}}\right)$.

The resulting curves for various energies and their dependence on $T_{\mathrm{o}}$ are shown in fig. 1 . As is evident the model predicts strong violation of scaling, which appears most strikingly near $x=0$, but is masked at larger $x$ by a cross-over phenomenon. Such a behaviour of course strongly contradicts the picture obtained by parton considerations.

In fig. 2 we show the corresponding curves using the full non-asymptotic level density [3] (eq. (1) with pions and kaons as secondaries). This confirms the picture previously obtained with the asymptotic formula. The difference between figs. 1(b) and 2 is mainly due to the presence of the kaons in the calculation for fig. 2.

As a consequence of the form of the asymptotic spectrum predicted by the SBM, it is strongly suggested to present experimental data in the form

$G(\omega) \equiv \frac{\omega}{\sigma_{\text {tot }} \cdot E} \cdot \frac{\mathrm{d} \sigma}{p^{2} \mathrm{~d} p}$

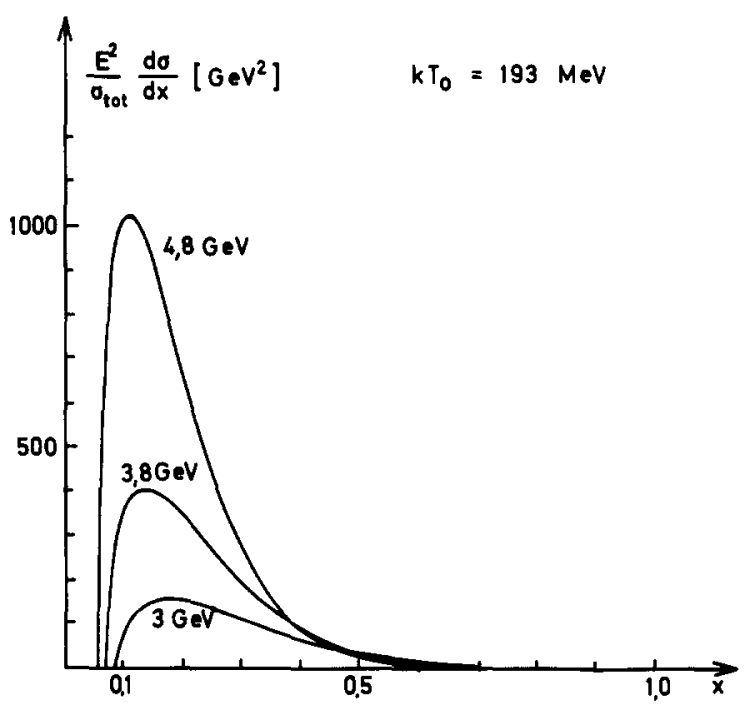

Fig. 2. Non-asymptotic predictions for the pion spectrum $\left(E^{2} / \sigma_{\text {tot }}\right)(\mathrm{d} \sigma / \mathrm{d} x)\left(\mathrm{e}^{+} \mathrm{e}^{-} \rightarrow\right.$ pions + kaons $)$ at $k T_{\mathrm{o}}=193 \mathrm{MeV}$ for $E=3,3.8$ and $4.8 \mathrm{GeV}$.

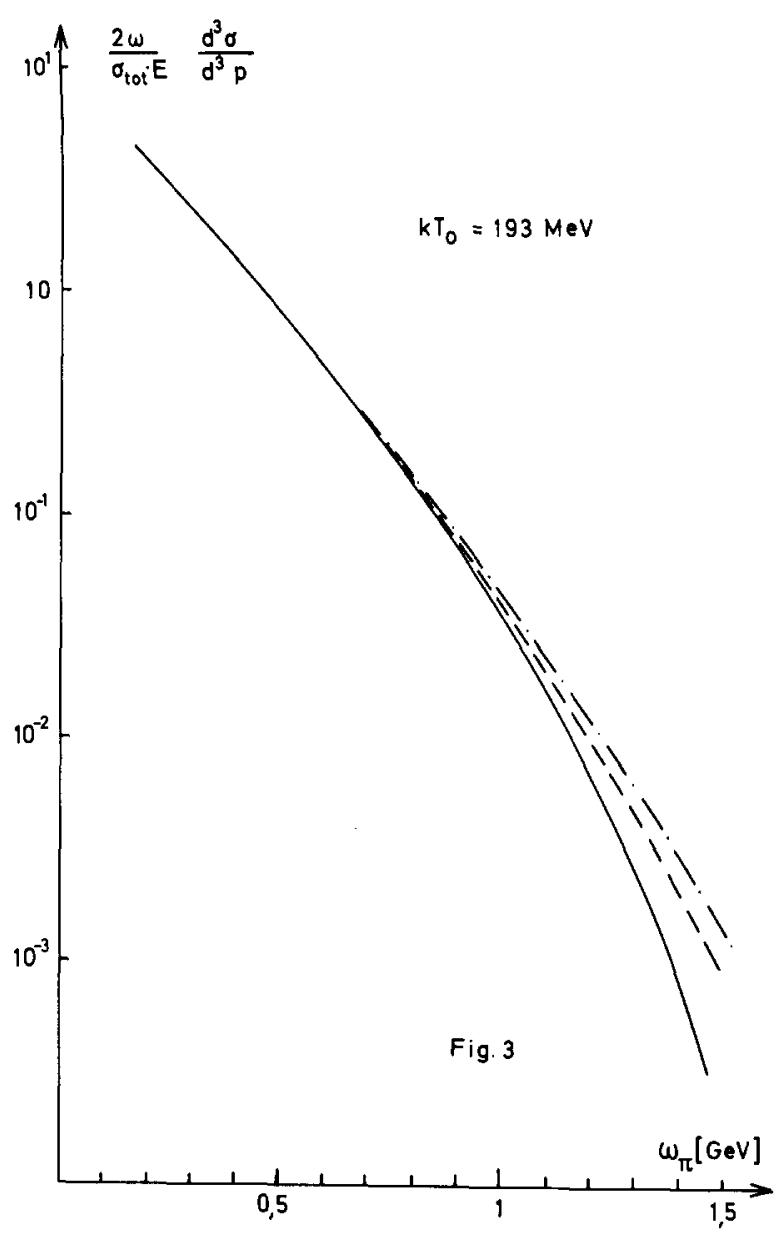

Fig. 3. Invariant spectrum $G(\omega)$ (eq. (8)) at $k T_{\mathrm{o}}=193 \mathrm{MeV}$ for $E=3 \mathrm{GeV}(-), 3.8 \mathrm{GeV})(--)$ and $4.8 \mathrm{GeV}(-\cdot-\cdot)$.

since in this representation all dependence on the incident energy should practically be removed. i.e. the SBM predicts $G(\omega)$ to be a universal function of the secondary energy $\omega$ for large $E$, as is demonstrated in fig. 3.

Since these features, as was repeatedly emphasized, provide a critical test of the SBM for $\mathrm{e}^{+} \mathrm{e}^{-}$annihilation, it will be extremely interesting to see whether the first positive indications for such behaviour [9] indeed persist in the future. If this should be the case, the concept of a fireball in the statistical bootstrap sense would for the first time receive direct empirical support.

We thank B. Nienhaber for his valuable help with the computer. 


\section{References}

[1] R. Hagedorn, Nuovo Cim. Suppl. 3 (1965) 147.

[2] S. Frautschi, Phys. Rev. D3 (1971) 2821.

[3] J. Engels, H. Satz and K. Schilling, Nuovo Cim. 17A (1973) 535 .

[4] M.I. Gorenstein et al., Bielefeld preprint Bi-73/12, Dec. 1973.

[5] J.D. Bjorken, Rapporteur's talk given at the Intern.
Symp. on Electron and photon interactions at high energies, Bonn, 1973.

[6] B. Alper et al., British-Scandinavian Collaboration, 16th Intern. Conf. on High energy physics, Batavia (USA) 1972.

[7] J. Yellin, Nucl. Phys. B52 (1973) 583.

[8] A. Bialas, invited talk given at the IVth Intern. Symp. on Multiparticle hydrodynamics, Pavia, 1973, CERN preprint TH 1745.

[9] B. Richter, talk presented at the 1973 Irvine Meeting. 